\title{
Kinetic Subpopulations Detected by Single-molecule Spectroscopy: Fundamental Property of Functional Nucleic Acids or Experimental Artefact?
}

\author{
Sebastian L. B. König§s, Danny Kowerko, and Roland K. O. Sigel ${ }^{\star}$ \\ \$SCS-Metrohm Foundation Award for best oral presentation
}

\begin{abstract}
Single-molecule spectroscopy allows the direct observation of conformational dynamics in individual biomolecules. Here, we describe how single-molecule Förster resonance energy transfer (smFRET) reveals heterogeneous kinetics in the EBS1*/IBS1* interaction, two RNA sequences that play an important role in group II intron mediated self-cleavage. Further examples of dynamic heterogeneity in functional nucleic acids are provided and the possible origins of this phenomenon are discussed.
\end{abstract}

Keywords: Heterogeneity · Group II introns · Memory effect · smFRET · Splicing

\section{Introduction}

Fluorescence-based methods have experienced unprecedented popularity to address biological questions. Detection of Förster resonance energy transfer (FRET) between a single pair of fluorophores attached to a biopolymer is of particular interest, because it provides a handle on measuring stochastic, time-dependent fluctuations in its structure. ${ }^{[1]}$ FRET is the distance-dependent, non-radiative energy transfer between two dipoles, typically the transition dipole moments of two fluorophores that are referred to as donor and acceptor. ${ }^{[2]}$ By estimating the interdye distance in real time, single-molecule FRET (smFRET) therefore allows the detection of intermediates along the folding pathway of biologically relevant molecules, as well as misfolded structures. As a consequence, it has been frequently used to study folding and function of catalytically active RNA molecules (ribozymes), regulatory elements of translation (riboswitches), and functional DNAs. ${ }^{[3,4]}$

In a typical smFRET experiment, nu-

*Correspondence: Prof. Dr. R. K. O. Sigel

Institute of Inorganic Chemistry

University of Zurich

Winterthurerstrasse 190

$\mathrm{CH}-8057$ Zürich

Tel.: +41446354652

E-mail: roland.sigel@aci.uzh.ch cleic acid samples are labelled with a fluorophore pair and a biotin moiety for subsequent immobilisation, even though smFRET studies can also be performed in solution, an approach that is routinely used to characterise proteins. ${ }^{[5,6]}$ Suitable dyes for FRET must be (i) bright (high quantum yield, high extinction coefficient $\varepsilon$ ), (ii) photostable (stable emission intensity, no blinking), (iii) small (minimal perturbation of the system under study), (iv) watersoluble and (v) easily attachable to the biomolecule of interest. ${ }^{[7]}$ Moreover, the fluorophore pair must display spectral overlap for FRET to occur. Cyanine dyes are the dominant choice as FRET probes in nucleic acid biophysics, but other dyes have also been successfully implemented. ${ }^{[8]}$ smFRET measurements are then carried out using either a confocal microscope or a total internal reflection fluorescence (TIRF) system (Fig. 1). Raw data are corrected for background noise, leakage of donor emission into the acceptor channel and direct excitation of the acceptor, so-called crosstalk. Finally, the corrected emission intensities are used to calculate FRET efficiency in order to probe intramolecular motion and intermolecular interaction. For further information on the implementation of smFRET and data analysis we direct the reader to the following excellent reviews. ${ }^{77,9]}$

The Yeast group II intron Sc.ai5gamma is one example where smFRET has been successfully employed to study ribozyme dynamics. ${ }^{[10-12]}$ These catalytic RNAs are several hundreds of base pairs in length and catalyse their own excision from the nascent messenger RNA molecule.[10] Recognition of the correct cleavage sites, i.e. the splice sites, is brought about by interaction of exon-binding sequences and intron-binding sequence (EBS1-3, IBS13). ${ }^{[13]}$ Here, a simple model system to study the influence of divalent cations on 5' splice site formation is proposed (Fig. 2a), followed by reporting and discussing heterogeneity observed with regard to its docking/undocking behaviour. ${ }^{[14]}$

\section{Materials and Methods}

\section{Single-molecule FRET Imaging}

The experimental design (Fig. 2a) involved surface immobilisation of the Cy3labelled EBS $1 *$ hairpin within a home-built microfluidic chamber via a streptavidinbiotin linkage. ${ }^{[4,13]}$ smFRET movies were recorded using a TIRF microscope under the following imaging conditions: $100 \mathrm{mM}$ $\mathrm{KCl}, 50 \mathrm{mM}$ MOPS, $1 \%$ D-glucose (w/V), $1 \mathrm{x}$ enzymatic oxygen scavenger solution (to prolong dye lifetime), and $1 \mathrm{mM}$ Trolox (for stable emission intensities), a protocol modified from Selvin and Ha. ${ }^{[8]}$ Furthermore, the imaging buffer contained $25 \mathrm{nM}$ Cy5-labelled IBS $1 *$ and variable $\mathrm{MgCl}_{2} / \mathrm{CaCl}_{2}$ concentration between 0 and $8 \mathrm{mM}$. Single-molecule clips were recorded over $6 \mathrm{~min}$ at $10 \mathrm{frames} / \mathrm{s}$ using an Andor 897 CCD camera (Lot Oriel, Romanel-surMorges, Switzerland). Oligonucleotides were ordered from Microsynth (Balgach, Switzerland) and chemicals were purchased purissum grade from SigmaAldrichs (Buchs, Switzerland). 


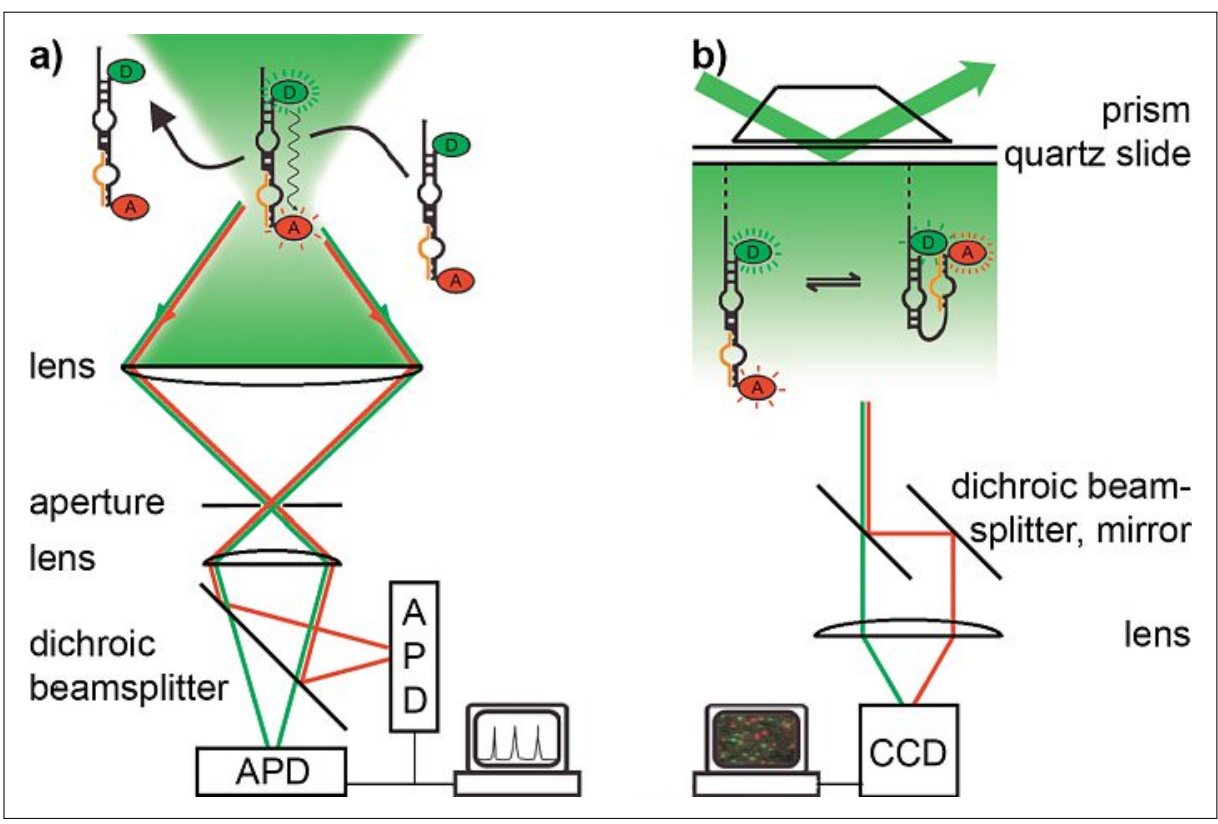

Fig. 1. Outline of single-molecule FRET microscopy systems. (a) Confocal microscopy. The donor fluorophore (D) is excited when the biomolecule traverses the focal volume (green area). FRET occurs when the acceptor dye $(A)$ is sufficiently close in space. Out-of-focus signal is rejected by an aperture, while in-focus bursts of fluorescence are detected upon spectral separation using avalanche photodiodes (APDs). It should be noted that photon multiplier tubes (PMTs) have also been used for detection, as well as the recently developed hybrid PMTs that decrease detection artefacts (photon afterpulsing, Picoquant GmbH, Berlin, Germany, personal communication). (b) TIRF microscopy. The incident laser beam reaches the surface at an angle that is larger than the critical angle, which leads to its total reflection and creates an evanescent wave reducing the excited volume to a thin sheet (green). Dye emission of surface-tethered biomolecules is spectrally separated and projected side-by-side onto a CCD camera, which allows to simultaneously observe hundreds of molecules over several minutes. Figure modified from ref. [1].

\section{Data Analysis}

Single-molecule clips were analysed using a home-written MATLAB-based software called SIRA (SIf Reader and Analyser), an approach detailed elsewhere.[9] Briefly, single fluorophore time trajectories were corrected for background noise and crosstalk (see above) prior to manual selection for anticorrelated emission profiles. Subsequently, the apparent FRET efficiency was calculated from corrected fluorophore emission time traces accord-

$$
F R E T=\frac{P C_{C y 3 \text { exc }}^{C y 5 \text { em }}}{P C_{C y 3 \text { exc }}^{C y 3 \text { ex }}+P C_{C y 3 \text { exc }}^{C y 5 \text { em }}}
$$

where $\mathrm{PC}$ refers to the photon counts due to $\mathrm{Cy} 3$ or $\mathrm{Cy} 5$ emission upon $\mathrm{Cy} 3$ excitation, respectively. ing to Eqn. (1),

\section{Results}

Single-fluorophore emission intensities fluctuated in a FRET-typical anticorrelated fashion (Fig. 2b,c), followed by a sudden drop in emission to zero (not shown). Such single-step photobleaching is important to rule out the prevalence of doubly-labelled RNA molecules. FRET time traces revealed the occurrence of two FRET states that were assigned to the docked (high FRET) and the undocked state (no FRET), as FRET can only occur when $\mathrm{Cy} 3$ and $\mathrm{Cy} 5$ are sufficiently close in space, typically in the range of $30-80 \AA$ depending on the FRET pair.

Interestingly, pronounced heterogeneity with regard to docking/undocking kinetics was observed in the presence of divalent cations (Fig. 2). Conventional approaches to determine rate constants (dwell time analysis) required three docking and three undocking rate constants to describe the experimental data recorded in the presence of divalent metal ions. ${ }^{[15]}$ Furthermore, rate constants associated with docking and undocking could not be unambiguously assigned to each other, which precluded the existence of three discrete kinetic subpopulations. Rather, rate constants seem to be continuously distributed over several orders of magnitude (Fig. 3 ), which suggests the coexistence of numerous (sub)structures.

Single-molecule studies rely on the principle of ergodicity, according to which the properties of billions of molecules can be reliably predicted by observing only a small number of them averaged over time (here: roughly 200). ${ }^{[16]}$ The averaged $\Delta G^{\circ}$ values associated with IBS $1 *$ docking determined from singlemolecule time trajectories were found to be in good agreement with the results of UV/vis thermal melting studies.[17] These

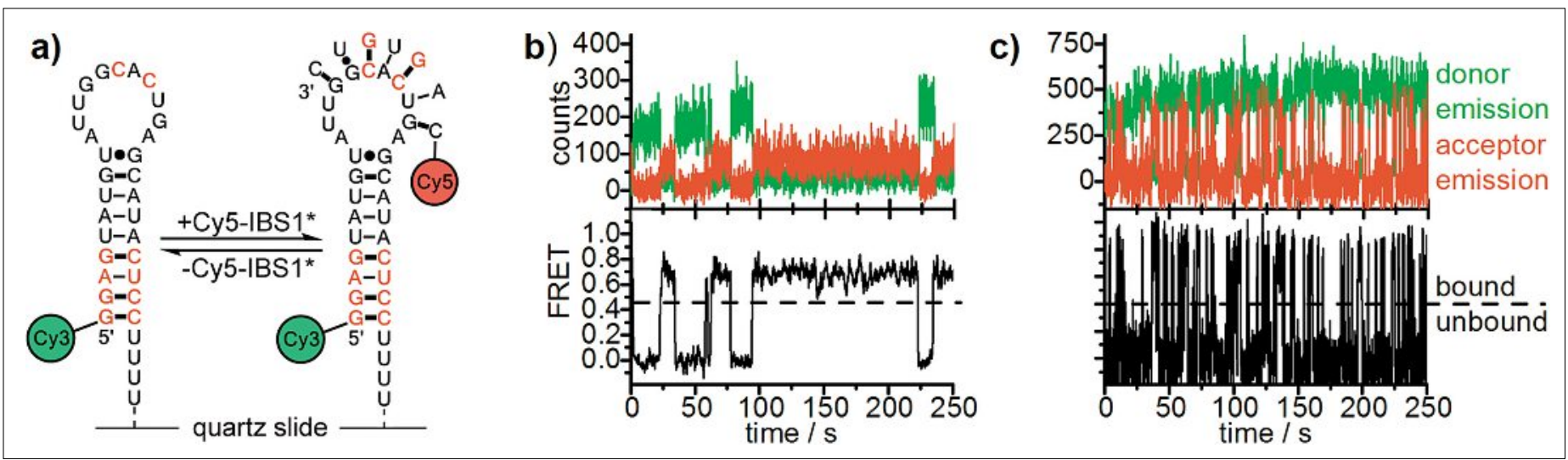

Fig. 2. Experimental design and representative data. (a) The EBS1 ${ }^{*}$ hairpin is Cy3-labelled and immobilised on the surface of a quartz slide. Docking of a Cy5-labelled IBS1* oligonucleotide is accompanied by a burst of Cy5 fluorescence and a decrease in Cy3 emission due to FRET. A: adenine, U: uracil, G: guanine, C: cytosine, interconnected via a phosphosugar backbone. Non-native bases are shown in red. ${ }^{[13]}$ (b, c) Two representative time trajectories recorded in the presence of $8 \mathrm{mM} \mathrm{CaCl}$, show clear differences in docking/undocking kinetics between individual molecules. Upper graphs: fluorophore emission over time reveals anticorrelated changes typical for FRET. Lower graphs: FRET over time shows fluctuations between a zero FRET state (undocked) and a high FRET state (docked). Figure modified from ref [14]. 


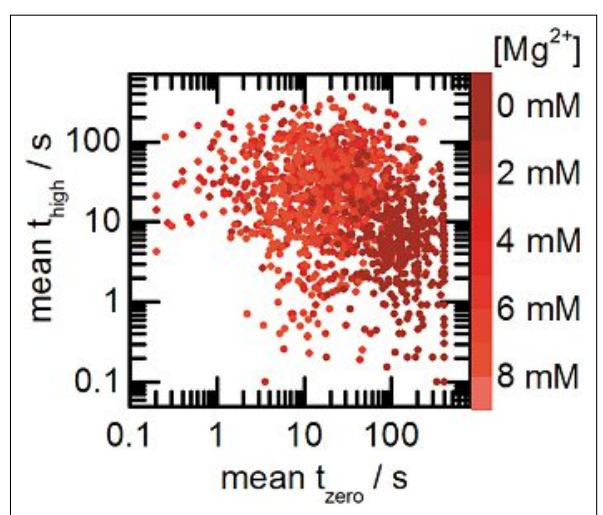

Fig. 3. Kinetic heterogeneity of EBS1*/IBS1* docking. The mean times spent in the docked $\left(t_{\text {high }}\right)$ and in the undocked state $\left(t_{\text {zero }}\right)$ are shown for individual EBS1* molecules at different $\mathrm{Mg}^{2+}$ concentrations. Figure modified from ref [14].

findings suggest that the complex docking/ undocking kinetics observed in the smFRET experiments are not an experimental artefact. Consequently, a novel method to describe the distribution of equilibrium constants was proposed and applied. As a comprehensive description would go beyond the scope of this article, the authors direct the interested reader to the original article. ${ }^{[14]}$

\section{Discussion}

At first, the complex kinetic behaviour observed for $\mathrm{EBS} 1 * / \mathrm{IBS} 1 *$ interaction may seem somewhat surprising given the simplicity of the experimental system. However, numerous examples of kinetic heterogeneities in nucleic acid folding have been precedented in single-molecule studies. Heterogeneity may be classified as static or dynamic, where static heterogeneity refers to the coexistence of stable subpopulations that do not interconvert ('memory effect'), while dynamically heterogeneous subpopulations interconvert during the time of observation. ${ }^{[18]}$

Heterogeneous folding kinetics have first been described in the hairpin ribozyme, which catalyses cleavage and ligation reactions in the replication cycle of the tobacco ringspot virus. ${ }^{[19]}$ In an analogous fashion to ESB1*/IBS1*, the hairpin ribozyme alternates between a docked and an undocked conformation and dynamics directly correlate with catalytic activity. While heterogeneity in cleavage kinetics had already been observed in bulk studies using a 'minimal ribozyme', i.e. a shorter version that is still catalytically active, Zhuang et al. could directly observe heterogeneity in structural dynamics between single hairpin ribozymes performing smFRET studies. ${ }^{[19,20]}$ While docking could be described with a single rate constant, undocking required a

Table 1. Kinetic heterogeneity of biologically relevant nucleic acids as revealed by single-molecule spectroscopy, in order of appearance in the text.

Biological model
5' splice site in group II
introns
Minimal hairpin ribo-
zyme
Native hairpin ribozyme

Tetrahymena group I
intron

Holliday junction

Human telomeric G-quadruplex

\section{Key findings}

3 docking rate constants, 3 undocking rate constants in the presence of $\mathrm{Mg}^{2+}$ or $\mathrm{Ca}^{2+}$

1 docking rate constant, 4 undocking rate constants, static undocking heterogeneity

Undocking rate constants span 3 orders of magnitude at low $\left[\mathrm{Mg}^{2+}\right]$, static undocking heterogeneity

Individual equilibrium constants vary by a factor of up to $300, \geq 6$ kinetic subpopulations, interconversion upon removal and re-addition of $\mathrm{Mg}^{2+}$

Partitioning of experimental data into five kinetic subspecies, interconversion upon removal and re-addition of $\mathrm{Mg}^{2+}$

Kinetic subspecies display either slow (min) or fast (s) structural rearrangements, interconversion between kinetic subgroups possible

References

[14]

total number of four exponential fit functions to satisfactorily describe the experimental data and the resulting rate constants spanned four orders of magnitude.[20] In addition, individual ribozymes were observed to dissociate with similar rate constants over long periods with less than $5 \%$ of interconversion within $3 \mathrm{~h}$ (static heterogeneity).[20] In a follow-up study, Ditzler and co-workers observed that the hairpin ribozyme migrates as two discrete bands on a non-denaturing gel electrophoretic shift assay, which differ in kinetics in subsequent smFRET experiments, thus confirming the longevity of the effect. ${ }^{[21]}$ Numerous examples of heterogeneous kinetics have followed and are summarized in Table 1. Please refer to ref. [26] for an excellent review on this topic.

\section{About the Origins of Heterogeneous Kinetics}

The origins of heterogeneous kinetics are likely to be diverse and they are subject of ongoing debate. One phenomenon known to bias smFRET studies is fluorophore photophysics, especially intersystem crossing to long-lived dark states ('blinking') and fluctuations in emission intensity. ${ }^{[27]}$ Even though the occurrence of undesired photophysical events can be greatly reduced by enzymatically removing molecular oxygen and upon addition of the vitamin $\mathrm{E}$ analogue Trolox, blinking appears to be a universal property of fluorophores. ${ }^{[27]}$ Therefore, the experimental design must be chosen carefully in order to clearly differentiate between changes in FRET caused by molecular motion and those caused by dye photophysics, which could otherwise contribute to the kinetic analysis. Unfortunately, this is not always possible depending on the biological system under study.

Probably the most fundamental feature shared by all functional nucleic acids is the fact that a precise fold must be adopted for functionality to be achieved. [26] As there is an almost infinite number of potentially available conformations (especially for long sequences), biomacromolecules may also adopt misfolded structures corresponding to local minima in the free energy landscape. ${ }^{[28]}$ Such kinetic traps are resolved in the living cell, e.g. by proteins, but they may accumulate in the artificial environment of the single-molecule studies that are frequently carried out under non-physiological conditions. [2] It is believed that kinetic heterogeneity is caused by the co-existence of several structures and a number of culprits have been proposed to this end: (i) Surface tethering. Immobilisation of biomolecules might alter their structure and hence their functionality. In fact, surface attachment has been shown to inactivate proteins. ${ }^{[29]}$ Nevertheless, this does not seem to hold true for RNA, as indirectly immobilised hairpin ribozymes encapsulated within lipid vesicles have been shown to behave like directly surface-tethered molecules. ${ }^{[22]}$ (ii) Chemical differences. Ditzler and co-workers excluded differences in molecular structure arising from chemical synthesis using high-resolution mass spectrometry.[21] As caveat it should be mentioned that possible mass-neutral modifications could not be detected. ${ }^{[26]}$ Exposure to UV-light during the purification process ('shadowing') as well as heating have recently been demonstrated to irrevers- 
ibly alter the chemically structure of RNA and thus leading to a broadened free energy landscape. ${ }^{[30]}$ (iii) Metal ion binding. Due to the polyanionic nature of the phosphate-sugar backbone, formation of nucleic acid structures is inextricably linked to the binding of metal ions. ${ }^{[31]}$ Here, magnesium ions are of particular interest because they are known to be specifically involved in folding and catalysis and owing to their bio-availability. ${ }^{[31]}$ However, compared to the affinity of metal ions to their specific binding sites in proteins $\left(K_{\mathrm{D}} \geq 10^{-6} \mathrm{M}\right.$ for surface-bound cations, $K_{\mathrm{D}}<10^{-7} \mathrm{M}$ for 'chaperoned' metal ions), their affinity to nucleic acid binding partners is lower by several orders of magnitude $\left(10^{-4} \mathrm{M} \leq K_{\mathrm{D}} \leq 10^{-2} \mathrm{M}\right) \cdot{ }^{[32,33]}$ Metal ion binding to nucleic acids is consequently of dynamic nature and the simultaneous occurrence of substructures with different cation binding patterns would be expected. Along this line, Solomatin et al. demonstrated that interconversion between kinetic subspecies observed for the Tetrahymena group I intron could be greatly accelerated in $\mathrm{Mg}^{2+}$ pulse experiments $\left(\mathrm{Mg}^{2+}\right.$ addition - removal - re-addition). ${ }^{[23]}$ Similar observations have recently been reported for the Holliday junction. ${ }^{[24]}$ In turn, cation-dependent experiments performed with the hairpin ribozyme suggest that the role of divalent cations is to stabilise the transition state by forming contacts that do not exist in the native conformation ( $\pi$ value analysis). ${ }^{[34-36]}$ Heterogeneities observed in group I introns and Holliday junction folding may therefore originate from differences in metal ion binding in the catalytically active fold, while this does not seem to hold true for the hairpin ribozyme. (iv) Molecular crowding. Macromolecules, especially proteins, occur at high concentrations inside a living cell. In fact, their concentration is so high that typically $5-40 \%$ of the total cell volume is physically occupied by these molecules. ${ }^{[37]}$ As a consequence, such crowding conditions will not only favour any reaction leading to increased volume (formation of compact conformations, aggregates, etc.), they will also reduce diffusion coefficients by a factor of up to 10 through increased viscosity (Stokes-Einstein equation). [37] Molecular crowding conditions have been mimicked in other areas of research through addition of polyethylene glycol (PEG), which led to dramatic shifts in the structural equilibrium. ${ }^{[38]}$ Future single-molecule studies should attempt to mimic the viscosity within a cell, which is likely to have an impact on the kinetic heterogeneity of nucleic acid samples.

\section{Conclusion}

The advent of single-molecule spectroscopy has provided a means to directly observe kinetic heterogeneities in biomolecular motion. Our recent report on heterogeneous docking/undocking behaviour of $\mathrm{EBS}^{*}$ and $\mathrm{IBS} 1 *$ takes its place among numerous other examples of complex kinetic behaviour unveiled by singlemolecule techniques. While we and others propose approaches to characterise molecular heterogeneity, ${ }^{[14,24]}$ discord persists over its possible origins that are likely to be diverse. Recent findings indicate, however, that kinetic heterogeneities in in vitro single-molecule studies are not an experimental artefact, given that the experimental design is chosen with care and suitable control experiment are carried out. The real challenge will be to find out whether kinetic heterogeneity exists in vivo. We believe that recent efforts in developing in vivo single-molecule techniques will provide novel proofs of principle and further our understanding of this fascinating phenomenon.

\section{Acknowledgements}

This work was supported by the European Research Council (ERC Starting Grant MIRNA $\mathrm{N}^{\circ} 259092$, to RKOS) and the Forschungskredit of the University of Zurich (57010302, SLBK), which is gratefully acknowledged. We are very thankful for further financial support related to our smFRET studies by the Swiss National Science Foundation and the Swiss State Secretariat for Education and Research (COST Action CM1105). SLBK thanks Prof. Dr. Eva Freisinger, University of Zurich, for valuable suggestions on the manuscript.

Received: February 27, 2013

[1] D. Rueda, N. G. Walter, J. Nanosci. Nanotechno. 2005, 5, 1990.

[2] S. L. B. König, P. Liyanage, R. K. O. Sigel, D. Rueda, RNA Biol. 2013, 10, 132.

[3] R. K. O. Sigel, A. M. Pyle, Chem. Rev. 2007, 107, 97.

[4] R. Zhao, D. Rueda, Methods 2009, 49, 112.

[5] M. B. Borgia, A. Borgia, R. B. Best, A. Steward, D. Nettels, B. Wunderlich, B. Schuler, J. Clarke, Nature 2011, 474, 662.

[6] L. Cardo, K. S. Karunatilaka, D. Rueda, R. K. O. Sigel, Methods Mol. Biol. 2012, 848, 227.

[7] R. Roy, S. Hohng, T. Ha, Nat. Methods 2008, 5 , 507.

[8] P. R. Selvin, T. Ha, 'Single-molecule techniques - a laboratory manual', Cold Spring Harbor Laboratory Press, Cold Spring Harbor, New York, USA, 2007.
[9] D. Kowerko, M. Hadzic, M. Heidernätsch, R. K. O. Sigel, 2013, submitted.

[10] M. Steiner, K. S. Karunatilaka, R. K. O. Sigel, D. Rueda, Proc. Natl. Acad. Sci. USA 2008, 105, 13853.

[11] M. Steiner, D. Rueda, R. K. O. Sigel, Angew. Chem. Int. Ed. 2009, 48, 9739.

[12] K. S. Karunatilaka, A. Solem, A. M. Pyle, D. Rueda, Nature 2010, 467, 935.

[13] D. Kruschel, R. K. O. Sigel, J. Inorg. Biochem. 2008, 102, 2147.

[14] D. Kowerko, S. L. B. König, L. Cardo, R. K. O. Sigel, 2013, submitted.

[15] X. Zhuang, L. E. Bartley, H. P. Babcock, R. Russel, T. Ha, D. Herschlag, S. Chu, Science 2000, 288, 2048.

[16] A. N. Kapanidis, T. A. Laurence, N. K. Lee, E. Margeat, X. Kong, S. Weiss, Acc. Chem. Res. $\mathbf{2 0 0 5}, 38,523$.

[17] S. L. B. König, J. L. Huppert, R. K. O. Sigel, A. C. Evans, 2013, submitted.

[18] A. N. Kapanidis, T. Strick, Trends Biochem. Sci. 2009, 34, 234

[19] N. G. Walter, J. M. Burke, Curr. Opin. Chem. Biol. 1998, 2, 24

[20] X. Zhuang, H. Kim, M. J. B. Pereira, H. P. Babcock, N. G. Walter, S. Chu, Science 2002, 296, 1473.

[21] M. A. Ditzler, D. Rueda, J. Mo, K. Håkansson, N. G. Walter, Nucleic Acids Res. 2008, 36, 7088.

[22] E. Tan, T. J. Wilson, M. K. Nahas, R. M. Clegg, D. M. J. Lilley, T. Ha, Proc. Natl. Acad. Sci. USA 2003, 100, 9308.

[23] S. V. Solomatin, M. Greenfield, S. Chu, D. Herschlag, Nature 2010, 463, 681

[24] C. Hyeon, J. Lee, J. Yoon, S. Hohng, D. Thirumalai, Nat. Chem. 2012, 4, 907.

[25] J. Y. Lee, B. Okumus, D. S. Kim, T. Ha, Proc. Natl. Acad. Sci. USA 2005, 102, 18938

[26] R. Zhao, D. Rueda, in 'Biophysics of RNA Folding (Biophysics for the Life Sciences)', Ed. R. Russell, Springer, New York, NY, 2012, in press.

[27] T. Ha, P. Tinnefeld, Annu. Rev. Phys. Chem. 2012, 63, 595 .

[28] R. T. Batey, R. P. Rambo, J. A. Doudna, Angew. Chem. Int. Ed. 1999, 2326

[29] R. Yasuda, T. Masaike, K. Adachi, H. Noji, H. Itoh, K. Kinosita Jr., Proc. Natl. Acad. Sci. USA 2003, 100, 9314.

[30] M. Greenfield, S. V. Solomatin, D. Herschlag, J. Biol. Chem. 2011, 286, 19872.

[31] E. Freisinger, R. K. O. Sigel, Coord. Chem. Rev. 2007, 251, 1834 .

[32] M. Pechlaner, R. K. O. Sigel, Met. Ions Life Sci. 2012, 1,1 .

[33] Z. Xiao, A. G. Wedd, Nat. Prod. Rep. 2010, 27, 768.

[34] L. E. Bartley, X. Zhuang, R. Das, S. Chu, D. Herschlag, J. Mol. Biol. 2003, 328, 1011.

[35] G. Bokinsky, D. Rueda, V. K. Misra, M. M. Rhodes, A. Gordus, H. P. Babcock, N. G. Walter, X. Zhuang, Proc. Natl. Acad. Sci. USA 2003, 100, 9302.

[36] D. Rueda, G. Bokinsky, M. M. Rhodes, M. J. Rust, X. Zhuang, N. G. Walter, Proc. Natl. Acad. Sci. USA 2004, 101, 10066.

[37] R. J. Ellis, A. P. Minton, Nature 2003, 425, 27.

[38] B. Heddi, A. T. Phan, J. Am. Chem. Soc. 2011, $133,9824$. 